\title{
Future directions in pharmacogenomics discovery in cardiovascular disease
}

\author{
Kevin A Friede ${ }^{*, 1}$ \& Deepak Voora ${ }^{1,2}$ \\ ${ }^{1}$ Division of Cardiology, Duke University, Durham, NC 27710, USA \\ ${ }^{2}$ Duke Center for Applied Genomics \& Precision Medicine, Duke University, Durham, NC 27708, USA \\ * Author for correspondence: kevin.friede@duke.edu
}

"6 A wealth of tools have been developed that can help pharmacogenomics realize the as-yet-unfulfilled promise of personalizing pharmacotherapy for patients with coronary artery disease (CAD). "”

First draft submitted: 3 January 2018; Accepted for publication: 5 January 2018; Published online: 23 March 2018

Keywords: coronary artery disease $\bullet$ pharmacogenomics

The late 1990s saw the development of new antiretroviral drugs for the treatment of HIV. One of these drugs, abacavir, had excellent clinical efficacy, but about $5 \%$ of patients to whom it was administered developed hypersensitivity reaction (HSR). In 2002, several research groups identified a link between HSR and the presence of MHC class I allele $H L A-B^{*} 5701$. At first, skin patch testing was used to confirm the presence of the HSR in patients who had reacted to abacavir, but this could not be done prospectively to inform drug choice, as patients needed to be immunologically primed to experience the HSR by having previously been exposed to abacavir. The landmark PREDICT-1 trial screened patients according to their HLA-B*5701 genotype and eliminated HSR in the study group [1], demonstrating the potential for this genetic information to be used as an effective screening tool. Because the HLA allele was a strong marker for toxicity with a high negative predictive value, its discovery was translated into quantitative and real-time PCR testing which could be performed in commercial laboratories [2,3]. HLA-B*5701 testing prior to initiation of abacavir is now a core recommendation in society guidelines for treatment of HIV [4].

The abacavir story demonstrates the great promise of pharmacogenomics in clinical medicine, and similar efforts have been adopted by others to develop screening algorithms in several areas of cardiovascular disease including, most notably, the use of the antiplatelet agent clopidogrel. The CYP2C19 enzyme, which helps to convert clopidogrel to its active state, has multiple variants that diminish the activity of the enzyme resulting in decreased levels of active metabolite and decreased efficacy in platelet inhibition [5]. The presence of these lossof-function alleles has been closely linked with an increased risk of death, myocardial infarction (MI) and stroke in clinical trials. However, for intermediate metabolizers who have only one loss-of-function allele, other clinical factors such as age and the presence of the metabolic syndrome (obesity, glucose intolerance, hypertension and lipid disorders) result in wide interindividual variability, which confounds the predicted clinical outcome. In addition, there are multiple additional well-documented genetic influences on the pharmacokinetic and pharmacodynamics properties of clopidogrel, including ABCB1, P2RY12, CYP2C9 and CES1, as well as other factors that affect underlying differences in platelet aggregation. Because of these intricacies, as well as the ready availability of alternatives such as prasugrel and ticagrelor, which circumvent defects in the CYP2C19 enzyme, CYP2C19 allele testing has not become the standard of care and has not been recommended (except in special cases) by the US FDA or the major cardiovascular professional societies.

It is likely that most of the pharmacogenetic variability in drug response is more complicated than the lowhanging fruit of abacavir hypersensitivity. Most pharmacogenetic findings to date relate to altered pharmacology (i.e., genetic variants in genes that affect drug metabolism, transport or elimination). Although these factors are important, variability in the underlying disease processes targeted by drug therapy likely explain more of the variation in drug response. A wealth of tools have been developed that can help pharmacogenomics realize the as-yet-unfulfilled promise of personalizing pharmacotherapy for patients with coronary artery disease (CAD). One

Future Medicine 
potential is to develop models based on the data from genome-wide data that use multiple inputs - several gainand loss-of-function alleles for a single gene, as well as multiple noncoding alleles across the genome - to define the predicted response to a given drug. Genome-wide models may be able to provide more information than single-gene analysis. However, CAD is a complex disease, and it is notable that multiple iterative genome-wide association studies (GWAS) for CAD have only been able to account for between 10 and $20 \%$ of CAD heritability [6]; performing similar analyses for drug effectiveness or adverse effects is likely to face the same hurdles and require much larger samples sizes than is typically feasible. One important study by Mega et al. created a genetic risk score for incident or recurrent CAD events based on GWAS findings and used this score to stratify patients according to how likely they were to benefit from statin therapy [7]. Such an approach could be considered for other drug classes as well as for other cardiovascular diseases: for example, by building genetic risk scores for hypertension and testing for response with diuretics; building scores for diabetes and testing for diabetes prevention from metformin in patients with insulin resistance; or building scores for atrial fibrillation and testing efficacy of anticoagulants for systemic embolism, for example. This would leverage the large investments in sample size and genetic findings from existing GWASs for complex diseases into predicting drug responses. As a result, developing a polygenic genetic risk score for the underlying disease can be used to fill in a missing gap in the current landscape of pharmacogenetic markers.

In addition to genome-wide risk scores, an alternative may be to explore drug response using a systems biology approach. Such a strategy would likely require the examination of multiple pathways (RNA, protein and metabolites) and modeling of drug response at the cellular, organ and whole-body level. Our group has developed a gene expression score that characterizes the response to aspirin using a profile of $62 \mathrm{mRNA}$ transcripts measured in peripheral blood [8]. The resultant aspirin response score is reproducible and not confounded by the presence of diseases such as $\mathrm{CAD}$ or diabetes, nor by concomitant medication use. In the area of metabolomics, a landmark study by Lewis et al. demonstrated that metabolomics techniques could be used to differentiate between a 'planned' MI due to elective alcohol septal ablation from spontaneous MI from CAD using blood samples [9]. A number of studies have used similar techniques to describe processes such as diabetic cardiomyopathy and to predict which patients are at risk for CAD [10,11]. Dynamic systems testing, in which a system is defined at baseline and then after some perturbation (for example, an oral glucose tolerance test or exercise testing to provoke myocardial ischemia [12]) could easily be adapted to define drug responses and to identify patients who may have an atypical or abnormal response. One could envision using this technique to define a 'normal' response profile to multiple classes of drugs - antiplatelet agents, statins or direct oral anticoagulants, for example - to define patients who are most likely to benefit. This could also be hypothesis-generating if it were able to define groups of patients who responded abnormally, providing insight into potential pathways into the mechanisms of such differences. This strategy could be used either to generate a new kind of biomarker (form), or to gain information about disease mechanisms (function) [13], as was demonstrated in a study of quantitative trait loci associated statin intolerance which identified a candidate mechanism of disease in a creatine synthesis pathway [14]. Because these strategies would be difficult to scale up for use in the clinic due to their reliance on advanced sample processing methods such as gas chromatography and mass spectrometry, simpler disease- or drug-specific testing would need to be developed once the fundamental candidate metabolites and pathways were defined for a particular clinical scenario [15].

It is clear that pharmacogenomics has not yet fulfilled its potential in the area of CAD and cardiovascular disease in general. However, we remain hopeful that some of the strategies we have proposed above can translate into real clinical benefit in the coming years. In the era of personalized medicine, we believe that pharmacogenomics will be a critical component of the arsenal for tailoring and improving medical therapies for patients with cardiovascular disease.

Financial \& competing interests disclosure

D Voora receives a research grant from AstraZeneca Pharmaceuticals, the NIH and the US Department of Defense. The authors have no other relevant affiliations or financial involvement with any organization or entity with a financial interest in or financial conflict with the subject matter or materials discussed in the manuscript apart from those disclosed.

No writing assistance was utilized in the production of this manuscript. 


\section{References}

1 Mallal S, Phillips E, Carosi G et al. HLA-B*5701 screening for hypersensitivity to abacavir. N. Engl. J. Med. 358(6), 568-579 (2008).

2 Russo CD, Lisi L, Lofaro A et al. Novel sensitive, specific and rapid pharmacogenomic test for the prediction of abacavir hypersensitivity reaction: HLA-B*57:01 detection by real-time PCR. Pharmacogenomics 12(4), 567-576 (2011).

3 Lalonde RG, Thomas R, Rachlis A et al. Successful implementation of a national HLA-B*5701 genetic testing service in Canada. Tissue Antigens 75(1), 12-18 (2010).

4 Aberg JA, Gallant JE, Ghanem KG, Emmanuel P, Zingman BS, Horberg MA. Primary care guidelines for the management of persons infected with HIV: 2013 update by the HIV medicine association of the infectious diseases society of America. Clin. Infect. Diseases 58(1), e1-e34 (2014).

5 Mega JL, Close SL, Wiviott SD et al. Cytochrome P-450 polymorphisms and response to clopidogrel. N. Engl. J. Med. 360(4), 354-362 (2009).

6 Consortium CD. A comprehensive 1000 genomes-based genome-wide association meta-analysis of coronary artery disease. Nat. Genet. 47, 1121 (2015).

7 Mega JL, Stitziel NO, Smith JG et al. Genetic risk, coronary heart disease events, and the clinical benefit of statin therapy: an analysis of primary and secondary prevention trials. Lancet 385(9984), 2264-2271.

8 Voora D, Cyr D, Lucas J et al. Aspirin exposure reveals novel genes associated with platelet function and cardiovascular events. J. Am. Coll. Cardiol. 62(14), 1267-1276 (2013).

9 Lewis GD, Wei R, Liu E et al. Metabolite profiling of blood from individuals undergoing planned myocardial infarction reveals early markers of myocardial injury. J. Clin. Invest. 118, 3503+ (2008).

10 Griffin JL, Atherton H, Shockcor J, Atzori L. Metabolomics as a tool for cardiac research. Nat. Rev. Cardiol. 8, 630 (2011).

11 Shah SH, Sun J-L, Stevens RD et al. Baseline metabolomic profiles predict cardiovascular events in patients at risk for coronary artery disease. Am. Heart J. 163(5), 844-850 (2012).

12 Van Der Greef J, Hankemeier T, McBurney RN. Metabolomics-based systems biology and personalized medicine: moving towards $\mathrm{n}=1$ clinical trials? Pharmacogenomics 7(7), 1087-1094 (2006).

13 Shah SH, Kraus WE, Newgard CB. Metabolomic profiling for the identification of novel biomarkers and mechanisms related to common cardiovascular diseases. Form Function 126(9), 1110-1120 (2012).

14 Mangravite LM, Engelhardt BE, Medina MW et al. A statin-dependent QTL for GATM expression is associated with statin-induced myopathy. Nature 502, 377 (2013).

15 Medina MW, Theusch E, Naidoo D et al. RHOA is a modulator of the cholesterol-lowering effects of statin. PLoS Genetics 8(11), e1003058 (2012) 
